\title{
AVALIAÇÃO DO GRAU FUNCIONAL DO ASSOALHO PÉLVICO EM UM GRUPO DE MULHERES DIABÉTICAS COM INCONTINENCIA URINÁRIA
}

\author{
Lariza Ragnini ${ }^{1}$ \\ Valdemira Santina Dagostin ${ }^{2}$ \\ Maria Tereza Brasil Zanini ${ }^{3}$ \\ Karina Cardoso Gulbis ${ }^{4}$ \\ Neiva Junkes Hoepers ${ }^{5}$ \\ Magada Tessmann 6
}

RESUMO: O presente estudo teve como objetivo avaliar o grau funcional do assoalho pélvico de mulheres diabéticas com Incontinência Urinaria (IU). Trata-se de uma pesquisa quantitativa, transversal, descritiva, de campo, desenvolvida com 2i mulheres diabéticas atendidas no Programa de Auto monitoramento Glicêmico da Clínica de Enfermagem. Os resultados demonstraram que a maioria das mulheres diabéticas, que tem idade média de 40 anos, com comorbidades, com Incontinência urinária de esforço, apresentam Grau 4 quando avaliado a força do assoalho pélvico. A avaliação do assoalho pélvico e o conhecimento precoce do grau de comprometimento deste, esta detecção precoce pode auxiliar o Enfermeiro a planejar ações de reabilitação do assoalho pélvico e consequente redução da IU.

Palavras-chave: Incontinência urinária. Diabetes Mellitus. Assoalho pélvico.

ABSTRACT: This study aimed to evaluate the functional degree of the pelvic floor of diabetic women with Urinary Incontinence (UI). This is a quantitative, cross-sectional, descriptive, field research, developed with 2I diabetic women assisted by the Nursing Clinic's Glycemic Self-Monitoring Program. The results showed that most diabetic women, who have an average age of 40 years, with comorbidities, with stress urinary incontinence, present grade

\footnotetext{
1 Graduanda em enfermagem pela Universidade do Extremo Sul Catarinense. E-mail: lary_ragnini@hotmail.com.

2 Enfermeira e docente na Universidade do Extremo Sul Catarinense (UNESC). Doutora em Ciências da Saúde pela Universidade do Extremo Sul Catarinense. E-mail: vsd@unesc.net.

3 Enfermeira e docente na Universidade do Extremo Sul Catarinense (UNESC). Especialização em Curso Micropolítica da Gestão e Trabalho em Saúde pela Universidade Federal Fluminense. E-mail: mbz@unesc.net.

${ }^{4}$ Enfermeira e docente na Universidade do Extremo Sul Catarinense (UNESC). Doutora em Ciências da Saúde pela Universidade do Extremo Sul Catarinense.E-mail: karina@unesc.net.

5 Enfermeira e docente na Universidade do Extremo Sul Catarinense (UNESC). Mestre em Ciências da Saúde pela Universidade do Extremo Sul Catarinense. E-mail: neivajun@unesc.net.

6 Enfermeira e docente na Universidade do Extremo Sul Catarinense (UNESC). Doutora em Ciências da Saúde pela Universidade do ExtremoSul Catarinense. E-mail: magada@unesc.net.
} 
4 when the pelvic floor strength is assessed. The assessment of the pelvic floor and early knowledge of the degree of impairment of this, this early detection can help nurses to plan actions to rehabilitate the pelvic floor and consequent reduction of UI.

Keywords: Urinary incontinence. Diabetes Mellitus. Pelvic floor

\section{INTRODUÇÃO}

O Diabetes Mellitus (DM) é uma doença crônica assinalada pela hiperglicemia e deficiência absoluta e/ou relativa de insulina, que intervém diretamente no metabolismo dos glicídios, proteínas, lipídios, água e vitaminas (FERREIRA; BARROS; BRAGA, 2016). Para Cândido et al., (2017) uma das complicações da Diabetes pode ser a incontinência urinária (IU) que é caracterizada pela perda de urina, causando incômodo para a mulher no contexto social e nas questões higiênicas, sendo classificada em cinco tipos: de urgência, de esforço, mista, contínua ou paradoxal. A etiologia da IU é multifatorial, porém nas pessoas idosas são mais frequentes, decorrentes das comorbidades, medicamentos ou deficiências funcionais que a senilidade acarreta (ROSA et al., 2019).

Segundo Braga et al., (2021) em 2018, a Sociedade Brasileira de Urologia (SBU) pontuou que mundialmente a IU se manifesta em por volta de 400 milhões de indivíduos, e consideram que no Brasil há cerca de io milhões de pessoas com algum dos cinco tipos de incontinência urinária, sendo que a prevalência maior ocorre nas mulheres, de diferentes idades ou etnias. Estudos de Salomé, Oliveira e Pereira (2016) revelou em uma a mostra de 910 mulheres, que 237 eram diabéticas, e a IU mais prevalente foram de esforço (IUE) e a mista foram em mulheres diabéticas.

A IU exerce diversos efeitos sobre as atividades diárias, intercâmbios sociais e percepção própria de saúde. As maiores dificuldades são relacionadas ao bem-estar social e mental, abrangendo problemas sexuais, isolamento social, baixa autoestima e depressão afetando de modo expressivo a qualidade de vida, com efeitos psicológicos, físicos, profissionais, sexuais e sociais (SABOIA et al., 2017). A musculatura do assoalho pélvico tem uma extraordinária função na sustentação dos órgãos pélvicos e abdominais e no mando da continência urinária e fecal, além de atuar na função sexual (MENDES et al., 2016).

A avaliação da força muscular do assoalho pélvico (FMAP) é importante para o cuidado, diagnóstico e tratamento de diversas situações de saúde da mulher. A FMAP pode ser avaliada em repouso ou em atividade, através da resistência e da contração muscular, por meio de exame ginecológico, utilizando-se métodos como: palpação digital vaginal, perineometria, ultrassonografia, eletromiografia, manometria e cones vaginais (DEDICAÇÃO et al., 2009).

Diante da temática "Incontinência Urinária em Diabéticas", o presente estudo teve como principal objetivo avaliar o grau funcional do assoalho pélvico de mulheres diabéticas com Incontinência urinária, atendidas na clínica escola de enfermagem UNESC.

\section{Metodologia}

O presente trabalho foi resultado de uma pesquisa quantitativa, transversal, descritiva e de campo, que apresentou como variáveis dependentes: grau de comprometimento do assoalho pélvico e independentes: incontinência urinária. O estudo foi desenvolvido na Clínica de Enfermagem da UNESC, no Programa de 
Automonitoramento glicêmico (PAMG), com um grupo de 2I mulheres diabéticas insulodependentes atendidas no PAMG durante o mês de maio de 2021. A seleção dos pacientes foi realizada conforme agenda do setor e aceitação dos pacientes.

Os critérios de inclusão foram: Ser mulher atendida no PAMG durante o mês de maio de 2021; aceitar participar da pesquisa mediante assinatura do Termo de Consentimento Livre e Esclarecido e de exclusão: Ser menor de idade.

O projeto foi submetido para análise do Comitê de Ética e Pesquisa com seres Humanos da UNESC com parecer de aprovação de número 46628721.3.oooo.oirg. Após aprovado seguiu o seguinte itinerário: Identificação das mulheres atendidas no PAMG; Seleção das mulheres atendidas no mês de maio de 2021 no PAMG; Avaliação do grau de funcionalidade do assoalho pélvico conforme Ortiz (1996).

Ortiz et al (1994) desenvolveram a avaliação funcional do assoalho pélvico (AFA) que foi graduada de o a 5 de acordo com a visualização da atividade contrátil dessa musculatura e a sensibilidade da palpação digital dessa contração. Os graus de força muscular podem ser classificados da seguinte forma: Grau o = sem função perineal objetiva nem mesmo à palpação; Grau I = função perineal objetiva ausente, reconhecida somente à palpação; Grau 2 = função perineal objetiva débil, contração fraca à palpação; Grau 3 = função perineal objetiva e resistência não opositora à palpação; Grau 4 = função perineal objetiva e resistência opositora não mantida mais do que cinco segundos à palpação; Grau 5 = função perineal objetiva e resistência opositora mantida mais do que cinco segundos à palpação.

Construção do banco de dados e discussão dos resultados. Todos os dados coletados foram digitados em arquivo do Microsoft Office Excel; analisados através da estatística descritiva, sendo gerados tabelas de frequência e gráficos das variáveis mais importantes do estudo. Todos os testes foram realizados com nível de significância de $5 \%$ e intervalo de confinça de $95 \%$.

\section{Resultados}

Foram avaliadas 21 mulheres diabéticas, das quais 63\% têm mais de 40 anos, I00\% apresentaram sintomas de Incontinência urinária e a maioria delas caracterizadas como de esforço. Foi feito o exame de avaliação da força do assoalho pélvico, onde pela introdução de dois dedos na vagina da mulher é avaliado o grau de força da contração voluntária da pelve. A preferência pelo uso da classificação funcional do assoalho pélvico utilizando-se a técnica proposta por Ortiz (1996) aconteceu pela simplicidade e rapidez da aplicação da técnica. A avaliação da contração voluntária é a chave para determinar a modalidade do tratamento, na escala o a 5, mostrando a ausência e normalidade da força muscular, respectivamente (ORTIZ, 1996), conforme é demonstrado na Tabela i:

Tabela I: Classificação funcional do assoalho pélvico de acordo com a contração voluntária

\begin{tabular}{|c|c|c|}
\hline Grau & Função subjetiva, visualização da contração em posição & À palpação \\
ginecológica & Ausente \\
\hline 0 & Ausente & Reconhecível \\
\hline I & Ausente & Reconhecível \\
\hline 2 & Débil & Sem resistência \\
\hline 3 & Presente & Com resistência <5” \\
\hline 4 & Presente & \\
\hline
\end{tabular}


(resistência não mantida)

Com resistência $>5$ "

5

Presente

(resistência mantida)

Fonte: Ortiz (1996) adaptado.

A partir desta avaliação, são expostos os resultados obtidos a partir do exame nas pacientes, como observado na tabela 2 .

Tabela 2: Grau de força do assoalho pélvico nas mulheres diabéticas com insuficiência urinária

Grau de força do assoalho pélvico nas mulheres diabética com UI

\begin{tabular}{|l|c|}
\hline Grau o & $23,8 \%$ \\
\hline Grau 2 & $9,5 \%$ \\
\hline Grau 3 & $14,3 \%$ \\
\hline Grau 4 & $52,4 \%$ \\
\hline
\end{tabular}

Observou-se que 23,8\% apresentam grau zero (nenhuma função à palpação), 9,5\% grau 2 (débil), I4,3\% grau 3 (presente sem resistência) e 52,4\% grau 4 (presente com resistência não mantida).

\section{Discussão}

O DM é uma doença metabólica marcada pelo aumento da glicemia e que pode ter como uma de suas complicações as IU, as quais, em mulheres diabéticas podem se apresentar de várias formas e com etiologias variadas (BROWN et al., 2006). A cistopatia diabética clássica é conceituada como uma redução da sensibilidade vesical e um aumento da capacidade vesical com hipoatividade do músculo detrusor. Apesar disso, a alta incidência de hiperatividade do detrusor na avaliação cistométrica de pacientes diabéticas aconselha que o acréscimo da atividade do detrusor é um atributo comum da cistopatia diabética, assim, a IU encontradas nas mulheres diabéticas podem variar da cistopatia diabética clássica à bexiga hiperativa, levando à incontinência urinária (GOMEZ, KANAGARAJAH, GOUSSE, 2oII).

Os três tipos mais frequentes de IU são: insuficiência urinária de esforço (IUE), quando há perda involuntária de urina com esforços como ao tossir ou espirrar; insuficiência urinária de urgência (IUU), quando a paciente acena o desejo repentino de urinar e não alcança controle sobre o músculo detrusor; e, insuficiência urinária mista (IUM), que é uma associação das duas formas citadas. Há consenso na literatura confirmando que a IU colabora para o surgimento de alterações psicológicas, nos relacionamentos pessoais, sexual e social, assim como, de alterações físicas e econômicas, influenciando negativamente a qualidade de vida dessas pessoas (SILVA et al., 2020). Podem surgir sintomas depressivos em mais de um terço das mulheres, onde a QV está comprometida pelos sinais e sintomas da IU (ROCHA; FREITAS; NERY, 20I8).

Vasconcelos e Ribeiro (2013) aludem que a avaliação funcional do assoalho pélvico permite a importância da competência, eficácia, tonicidade e resistência da contração voluntária desta musculatura, aceitando necessidade da adequação do planejamento terapêutico de acordo com a funcionalidade de cada paciente, trazendo maiores 
oportunidades de sucesso terapêutico.

Uma pesquisa desenvolvida por Frota (2016) no Ceará, que avaliou o grau da Força de avaliação funcional do assoalho da pelve (AFA), descrito por Ortiz em 1996, por meio do toque vaginal bidigital, com a mulher em posição para exame clinico, apresentaram resistência muscular diminuída (endurance e repetition), o que representa dificuldade em manter a contração. Este resultado corrobora com os resultados encontrados na presente pesquisa onde foi utilizada a mesma técnica, e mostrou $28,44 \%$ em grau o, ou seja, sem nenhuma função perineal, nem a palpação, 9,5\% grau 2 e $14,3 \%$ grau 3, representando resistência muscular diminuída e 52,4\% Grau 4 (presente com resistência não mantida).

A diabetes é uma doença metabólica, que pode produzir danos a longo prazo, como perda ou falha de função em vários órgãos, como os rins, além de vasos sanguíneos, olhos e nervos. Pode levar a quadros de infecção do trato urinário, hipertensão, anormalidades do metabolismo das lipoproteínas, retinopatia com perda de visão ou mesmo insuficiência renal (COSTA et al., 2020).

Cândido et al. (2017) relata que o exame pélvico é recomendado quando os achados, como a detecção de uma massa pélvica, alterariam a intervenção planejada ou influenciariam a seleção do tratamento. Em mulheres pós-menopausa, deve ser identificada a atrofia vaginal, que pode ser tratada efetivamente com estrogênio vaginal. Deve ser avaliado ainda, a integridade e a função muscular do assoalho pélvico durante o exame pélvico bidigital.

Casarin, Frigo e Gasparetto (2015) dizem que a IU associada a retenção crônica de urina é caraterizada pela perda involuntária contínua de urina associada a um incompleto esvaziamento da bexiga, que ocorre com maior frequência em idosos em estado de saúde mais precários, que referem frequência e urgência, sintoma de jato urinário fraco, micção incompleta e frequentes ou constantes pequenas perdas de urina.

De acordo com Rosa et al., (2019), a Internacional Continence Society (ICS) estima que uma a cada três pessoas que sofrem de incontinência sentem-se compelidas em falar sobre o assunto, convivendo com a situação por muitos anos, sem buscar auxilio, e avaliando a situação como normal. Tal situação, entretanto, podem disfarçar diversos aspectos da vida, não só o físico, como também o social, psicológico, ocupacional, doméstico e sexual.

Nascimento et al., (2020) aludem que a assistência de enfermagem elucubra a sustentação das práticas de saúde necessárias no cotidiano das pessoas, avaliando que o cuidar é uma das ferramentas do processo de trabalho que o enfermeiro prepara para aplicação do conhecimento técnico-científico, imperioso à assistência e aperfeiçoamento das suas ações. Assim, a assistência contribui verdadeiramente para que o exercício profissional do enfermeiro seja visto pela sociedade como arte do cuidar, deslocando-a para ciência que aponta para uma metodologia própria por meio do saber técnico-científico. Analisando isso, os profissionais de saúde alargam suas práticas a partir de capacidades construídas por meio de um processo de formação que tem por base a capitalização, desenvolvimento de conhecimentos e de tecnologias.

Schimidt et al. (2016) conceituam estomaterapia, como uma especialidade da prática de enfermagem, exclusiva do enfermeiro estabelecida, mundialmente pelo Conselho Mundial de Estomaterapia, a partir de 1980, voltada para o cuidado de pessoas com estomias, feridas agudas e crônicas, fístulas, drenos, cateteres e incontinências anais e o especialista é denominado Estomaterapeuta (ET).

A Resolução do COFEN 358/2009 (COFEN, 2009) dispõe sobre a sistematização de enfermagem e a implantação do processo de enfermagem em ambientes públicos ou privados, em que acontece o cuidado profissional de enfermagem. A partir do Art. $\mathrm{I}^{\circ} \S 2^{\circ}$ 
descreve as cinco etapas correlacionadas: I Coleta de dados (ou histórico de enfermagem; 2. Diagnóstico de enfermagem; 3. Planejamento; 4. Implementação; 5. Avaliação de enfermagem.

Uma das etapas do processo de enfermagem é coleta de dados, exame físico, diagnóstico de enfermagem, no qual consegue-se avaliar e identificar fatores contribuintes e reversíveis, bem como no planejamento, determinar estratégias apropriadas para minimizar e/ou solucionar a IU.

Monteiro, Fonseca e Silva Filho (2012) relatam que o exame urodinâmico (EU) é o método propedêutico de eleição para análise funcional do trato urinário inferior (TUI), que permite avaliar o comportamento vesical durante seu enchimento, e esvaziamento e, consequentemente, obter uma amostra do ciclo de micção do paciente. Além das informações que podem ser obtidas através do EU, alguns fatores como uso dos cateteres uretral e retal, infusão de soro fisiológico em poucos minutos e urinar na frente a estranhos, podem intervir nos resultados obtidos, assim, é decisivo correlacionar os achados urodinâmicos com a história clínica, o diário miccional e o exame físico.

Um parecer da ordem dos enfermeiros de Lisboa (2018), menciona que em um contexto de atuação multiprofissional, e de acordo com Artigo $9^{\circ}$, ponto I, 2 e 3 do Regulamento do Exercício Profissional dos Enfermeiro (REPE), enquadram-se dois tipos de intervenções: Intervenções Independentes: são as indicadas por outros técnicos da equipe, onde o Enfermeiro tem a reponsabilidade pela implementação técnica da intervenção. Intervenções autônomas: são as iniciadas pela prescrição do enfermeiro que é responsável pela prescrição da intervenção e implementação de ambas. Nestas duas intervenções apontadas anteriormente, os enfermeiros têm autonomia de decidirem a sua implementação, tendo como base os conhecimentos que detém, a identificação da problemática do paciente, os benefícios, os riscos e os problemas potenciais que podem advir da implementação, atuando da melhor forma diante do interesse das pessoas assistidas (LISBOA, 2018).

O estudo urodinâmico, é um exame que necessita de equipamento especial, qualificação para realização e interpretação, tem duração em torno de 30 a 60 minutos e é indolor. Consiste na introdução de uma sonda na bexiga através da uretra e outra no reto. Estas sondas têm sensores que medem a pressão. $O$ exame está dividido em três componentes: o primeiro consiste na fase de enchimento vesical lento, com Soro fisiológico (através do cateter) e concomitante avaliação das pressões intravesical e intrabominal (cateter retal) e na mensuração de eventual fluxo urinário (urofluxômetro); posteriormente, na fase miccional, onde é solicitado para o paciente urinar, é avaliado as curvas de pressão/fluxo, ou seja, a relação entre as pressões intravesicais e o fluxo urinário; no terceiro componente, denominado de perfilometria é onde se avalia a pressão de encerramento do esfíncter uretral, através de um transdutor instalado na extremidade de um cateter que percorre a uretra à medida que é retirado. $O$ estudo urodinâmico é realizado pelo médico urologista, trata-se de uma intervenção interdependente, responsabilizando-se o enfermeiro pela tomada de decisões e pelos atos que pratica ou delega (MONTEIRO; FONSECA; SILVA FILHO, 2012).

Schimidt et al. (2016) em parceria com a SOBEST organizaram a obra intitulada "Intervenções nas áreas de abrangência da estomaterapia, identificando Diagnósticos de Enfermagem”, Classificação da Sociedade Internacional de Continência (ICS), classificação das intervenções de enfermagem (NIC), profissional habilitado e classificação dos resultados esperados (NOC), apresenta-se na Figura I a seguir, o quadro que demonstra que 
o enfermeiro estomaterapeuta é apto para a realização de estudo urodinâmico:.

Figura r: Diagnósticos de enfermagem, classificação da ICS

\begin{tabular}{|c|c|c|c|c|}
\hline $\begin{array}{c}\text { Diagnóstico de } \\
\text { Enfermagem } \\
\text { (NANDA 2012 - 2014) }\end{array}$ & $\begin{array}{l}\text { CLASSIFICAÇÃO } \\
\text { ICS (Sociedade } \\
\text { Internacional de } \\
\text { Continência) }\end{array}$ & $\begin{array}{c}\text { INTERVENÇŌES } \\
\text { (Códigos NIC - } 5^{a} \text { edição - 2010) }\end{array}$ & $\begin{array}{l}\text { Profissional } \\
\text { Habilitado }\end{array}$ & $\begin{array}{l}\text { Resultados Esperados } \\
\text { (NOC - } 4^{\mathrm{a}} \text { edição - 2010) }\end{array}$ \\
\hline $\begin{array}{l}00016 \text { - Eliminação } \\
\text { urinária prejudicada } \\
00017 \text { - Eliminação } \\
\text { urinária por pressão } \\
00018 \text { - Incontinência } \\
\text { urinária reflexa } \\
00019 \text { - Incontinência } \\
\text { urinária de impulso } \\
00020 \text { - Incontinência } \\
\text { urinária funcional } \\
\text { 00021 - Incontinência } \\
\text { urinária total } \\
\text { 00023 - Retenção } \\
\text { urinária }\end{array}$ & $\begin{array}{l}\text { Disfunção } \\
\text { neurológica do trato } \\
\text { urinário inferior, } \\
\text { Hiperatividade } \\
\text { detrusora idiopática, } \\
\text { Cistite intersticial, } \\
\text { Cistite actínica, } \\
\text { Sindrome da dor } \\
\text { vesical, Incontinência } \\
\text { urinária de esforço, } \\
\text { Incontinência urinária } \\
\text { mista, Prolapso } \\
\text { de órgão pélvico, } \\
\text { Enurese noturna }\end{array}$ & $\begin{array}{l}\text { Realizar Exame Urodinâmico, } \\
\text { quando integrado à equipe } \\
\text { de cuidado a pacientes } \\
\text { incontinentes, desde que } \\
\text { obtenha os pré-requisitos } \\
\text { técnico-cientificos. } \\
\text { Código NIC } \\
0610 \text { - Cuidados na IU } \\
0620 \text { - Cuidados na retenção urinária }\end{array}$ & $\begin{array}{l}\text { Enfermeiro } \\
\text { Estomaterapeuta }\end{array}$ & $\begin{array}{l}\text { Controle da } \\
\text { eliminação de urina } \\
\text { Armazenamento e } \\
\text { eliminação de urina }\end{array}$ \\
\hline
\end{tabular}

Schimidt et al., (2016).

A reabilitação é definida como um processo dinâmico, que envolve o paciente, o cuidador, os familiares e a equipe multiprofissional com a finalidade de prevenir, retardar e compensar a perda de uma função, além de recuperar, melhorar e manter uma função atual, que nos dias de hoje, observa-se uma expansão da necessidade de cuidados de reabilitação, sendo preciso a ampliação do mercado de trabalho em reabilitação multiprofissional (WHO, 20II).

Segundo Honório (2006) a terapia comportamental, uma das estratégias de reabilitação, em particular, é um método não invasivo de reabilitação do assoalho pélvico na IU, de baixo risco, pouco dispendioso e que pode compor uma estratégia de tratamento efetiva e conservadora na recuperação das funções fisiológicas, causando uma melhora da musculatura do assoalho pélvico. Esta terapia, consiste em estimular modificações comportamentais e mudanças dos hábitos da mulher com IU que possam contribuir para piorar ou causar episódios de perdas urinárias, associando a isso um retreinamento da bexiga. O ideal é a participação de uma equipe multiprofissional, que possa dar orientações necessárias, incluindo alteração de hábitos alimentares, como minimização da ingestão de cafeinados, excesso de líquidos antes de dormir, frutas ácidas, achocolatados e refrigerantes, sendo estes considerados produtos irritantes vesicais e podem de certa forma agravar os episódios de perdas urinárias, sobretudo, nas situações de urge-incontinência.

Assim, a literatura aponta também, intervenções de enfermagem, que podem auxiliar no diagnóstico e controle da perda urinária contribuindo para melhorar a qualidade vida dessas pessoas (CÂNDIDO et al., 2017; SILVA et al., 2020; NASCIMENTO et al., 2020). Segundo Caldas et al., (2010) e também Silva e D'Elboux (2012), inúmeras terapêuticas são utilizadas pelo enfermeiro estomaterapeuta na reabilitação da IU, dentre elas, o uso de cones vaginais, exercícios perineais, exercício de Kegel, dentre outros. 


\section{CONCLUSÕES}

Este estudo alcançou seus objetivos à medida que identificou que mulheres diabéticas com incontinência urinária, tem faixa etária de 40 anos ou mais, caracterizadas como de esforço, $23,8 \%$ apresentam grau zero de força de assoalho pélvico.

A IU é nos dias atuais um problema de Saúde Pública, promovendo a segregação social, através do isolamento das mulheres (neste caso diabéticas), do convívio em grupos decorrente da perda urinária e odor que eventualmente podem causar. A atenção a esta população deve ser interdisciplinar e integral considerando que os agentes causais que podem intensificar a IU e as complicações que a mesma pode afetar seja na saúde mental como nas questões socioeconômicas.

Sugerimos que este estudo seja reaplicado com uma amostra maior de mulheres diabéticas que sofrem de IU, sendo realizados urocultura com antibiograma, avaliação nutricional, risco de ansiedade e depressão e avaliação de qualidade de vida e, se, possível, estudo urodinâmico, permitindo assim, o fornecimento de maiores subsídios para planejamento de ações multidisciplinares na promoção, prevenção e reabilitação desse público alvo.

Fica ainda, como sugestão, que nesta proposta de estudo ampliado, sejam inclusos acadêmicos de graduação das múltiplas áreas da saúde e da pós-graduação em estomaterapia já existente na Instituição. Por fim, a última sugestão é que seja construído ou formado um ambulatório específico de estomaterapia, para neste caso, atuar no diagnóstico e reabilitação pélvica para IU (e fecal), com protocolos de atendimentos e com suporte multiprofissional.

\section{REFERÊNCIAS}

BRAGA, Francisca das Chagas Sheyla Almeida Gomes et al. Perfil de pacientes com incontinência urinária em um ambulatório de hospital universitário. Revista Estima, São Paulo, volume i9, número I, e072I 202I.

BROWN, Jeanette S. et al. Prevalence and risk factors for urinary incontinence in women with type 2 diabetes and impaired fasting glucose: findings from the National Health and Nutrition Examination Survey (NHANES) 2001-2002. Diabetes Care, volume 29, número 6, página1307-1312, 2006.

CALDAS, Célia Pereira et al. Terapia comportamental para incontinência urinária da mulher idosa: uma ação do enfermeiro. Texto Contexto Enfermagem, Florianópolis, volume 19, número 4, pagina 783-788, 2010.

CÂNDIDO, Fernando José Leopoldino Fernandes et al. Incontinência urinária em mulheres: breve revisão de fisiopatologia, avaliação e tratamento. Visão acadêmica, Curitiba, volume I8, número 3, 2017.

CASARIN, Naiára; FRIGO, Leticia Fernandez; GASPARETTO, Andriele. O diabetes mellitus nas disfunções pélvicas femininas. Fisioterapia Brasil, volume I6, número I, 2015.

COFEN, Conselho Federal de Enfermagem. Resolução COFEN - 358/2009. Disponível em: 
〈http://www.cofen.gov.br/resoluo-cofen-3582009_4384.html〉.

COSTA, Heloneida Maria Leoncio Mahle et al. Associação entre neuropatia diabética e sintomas de trato urinário inferior nas pessoas com diabetes mellitus: revisão integrativa. Revista Eletrônica Acervo Saúde, volume 12, número I2, 2020.

DEDICAÇÃ O, Anny Caroline et al. Comparação da qualidade de vida nos diferentes tipos de incontinência urinária feminina. Brazilian Journal of Physical Therapy, volume 13 , número 2, 2009.

FERREIRA, Renata Carneiro; BARROS, Caroline Espíndola de; BRAGA, Ariane Leal. Perfil de infecção urinária associada à taxa de glicemia alterada. Revista Brasileira de Análises Clínicas, volume 48, número 4, página 346-51, 2016.

FROTA, Isabella Parente Ribeiro. Função do assoalho pélvico e qualidade de vida em mulheres na pós-menopausa com e sem disfunção do assoalho pélvico. 2016. 82 folhas. Dissertação (Mestrado em Cirurgia) - Universidade Federal do Ceará. Fortaleza: UFC, 2016.

GOMEZ, Cristopher S.; KANAGARAJAH, Prashhant; GOUSSE, Angelo E. Bladder dysfunction in patients with diabetes. Current Urology Reports, volume 12, número 2, página 419-26, 2011.

HONÓRIO, Melissa Orlandi. Educação para o autocuidado: uma alternativa de assistência de enfermagem ao adulto e idoso com incontinência urinária. 2006. Dissertação (Mestrado em Enfermagem) - Universidade Federal de Santa Catarina. Florianópolis: UFSC, 2006.

LISBOA, Portugal. Regulamento no․ 372/2018. Diário da República Eletrônico - DRE. Disponível em: <https://dre.pt/pesquisa/-/search/II5522772/details/normal?1=I $>$.

MENDES, Edilaine de Paula Batista et al. Força muscular do assoalho pélvico em primíparas segundo o tipo de parto: estudo transversal. Revista Latino-Americana de Enfermagem, volume 24, 2016.

MONTEIRO, Marilene Vale de Castro; FONSECA, Andrea Moura Rodrigues Maciel da; SILVA FILHO, Agnaldo Lopes. Valor do estudo urodinâmico no tratamento da incontinência urinária. Feminina, volume 40, número 3, página 135-139, 2012.

NASCIMENTO, Thamires Souza do et al. Atuação do enfermeiro na atenção básica diante do manejo da incontinência urinária feminina: uma revisão integrativa. Brazilian Journal of Health Review, volume 3, número 6, 2020.

ORTIZ, Contreras et al. Evaluación funcional del piso pelviano femenino (classificación funcional). Boletim de la Sociedad Latino Americana de Uroginecologia y Cirurgia Vaginal, volume I, número I, pagina 5-9, 1994.

Ortiz, Contreras et al. Dinâmica de la Disfuncíon Parineal de Classificacion. Boletim de la Sociedade Latino Americana de Urologia Y Cirurgia Vaginal, volume I, pagina 7-9, 1996. 
ROCHA, Luiza Assis; FREITAS, Paulo Fontoura; NERY, Luisa Aguiar da Silva. Fatores associados à incontinência urinária em mulheres atendidas na Policlínica Municipal de Palhoça. 2018. 2I folhas. Artigo Científico - Universidade do Sul de Santa Catarina. Florianópolis: Unisul, 2018.

ROSA, Maria Inês et al. Prevalência e fatores associados à incontinência urinária em mulheres diabéticas insulinodependentes do município de Criciúma/SC. Revista da AMRIGS, Porto Alegre, volume 63, número 4, página 407-413, 2019.

SABOIA, Dayana Maia et al. Impacto dos tipos de incontinência urinária na qualidade de vida de mulheres. Revista da Escola de Enfermagem da USP, São Paulo, volume 5I, 2017.

SALOMÉ, Geraldo Magela; OLIVEIRA, Thaís Fátima de; PEREIRA, Wesley Alexandre. $O$ impacto da incontinência urinária na autoestima e autoimagem de pacientes diabéticos. Estima - Brazilian Journal of Enterostomal Therapy, volume 14, número 3, 2016.

SCHIMIDT, Fernanda Mateus Queiróz et al. Intervenções nas áreas de abrangência da estomaterapia. Associação Brasileira de Estomaterapia: estomias, feridas e incontinências SOBEST. Lorena: Instituto Dona Teresa, 2016.

SILVA, Aurenice Gomes da et al. Incontinência urinária em mulheres: fatores de risco segundo tipo e gravidade. Cogitare Enfermagem, volume 25, 2020.

VASCONCELOS, Elaine Cristine Lemes Matheus; RIBEIRO, Aline Moreira. Força e função muscular do assoalho pélvico: como avaliar? Fisioterapia Brasil, volume i4, número 6, página 469-473, 2013.

WHO, World Health Organization. Use of Glycated Haemoglobin (HbAic) in the Diagnosis of Diabetes Mellitus. 2011. Disponível em: $\langle$ https://www.who.int/diabetes/publications/report-hbaic_20II.pdf〉. 